\title{
Psychopathologic Profiles and Clusters in Tertiary Clinic Referred Patients with Adult Attention Deficit Hyperactivity Disorder: A Person-Centered Analysis
}

\author{
Yunhye Oh${ }^{1}$, Hyewon Park ${ }^{2}$, Seonwoo Kim ${ }^{3}$, Hye Seung Kim³, \\ Yoo-Sook Joung ${ }^{4}$, Kyung Sue Hong ${ }^{4}$, and Ji Hyun Baek ${ }^{4}$ \\ ${ }^{1}$ Department of Child Adolescent Psychiatry, National Center for Mental Health, Seoul, Republic of Korea \\ ${ }^{2}$ Samsung Biomedical Research Institute, Seoul, Republic of Korea \\ ${ }^{3}$ Center for Biomedical Statistics, Research Institute for Future Medicine, Samsung Medical Center, Seoul, Republic of Korea \\ ${ }^{4}$ Department of Psychiatry, Sungkyunkwan University School of Medicine, Samsung Medical Center, Seoul, Republic of Korea
}

Psychiatry Investig 2021;18(4):304-311

https://doi.org/10.30773/pi.2020.0331

1. In the original published version of this article, references [16] and [36] were duplicated.

Reference [16] of reference section should be replaced by:

[16] Barkley RA. Attention-Deficit/Hyperactivity Disorder. Guilford Publications, 2006.

2. References [40] and [47] were duplicated.

1) Reference [47] of reference section should be removed.

Reference [48],[49],[50],[51], and [52] should be renumbered [47],[48],[49],[50],and [51], respectively.

2) The following citations in the 'Discussion' should be changed:

[47] replaced by [40].

[48] replaced by [47].

[49] replaced by [48].

[50] replaced by [49].

[51] replaced by [50].

[52] replaced by [51].

(a) This is an Open Access article distributed under the terms of the Creative Commons

Attribution Non-Commercial License (http://creativecommons.org/licenses/by-

nc/4.0) which permits unrestricted non-commercial use, distribution, and reproduc-

tion in any medium, provided the original work is properly cited. 